\title{
La producción de textos escritos en el aprendizaje de las ciencias. Bases para un programa de investigación
}

\author{
Juan A. Lloréns y M. ${ }^{a}$ del Carmen de Jaime
}
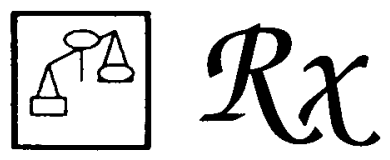

Podemos admitir que entre el profesorado de Educación Secundaria existe una opinión generalizada acerca de las numerosas dificultades y carencias que sus alumnos presentan en el uso oral y escrito del lenguaje en las diferentes disciplinas. Asimismo, los problemas linguiisticos constituyen uno de los aspectos principales de muchas de las concepciones alternativas y erróneas identificadas en la investigación educativa. El artículo pretende fundamentar teóricamente estos problemas para establecer una clasificación detallada de los mismos que sirva de base a la propuesta de un programa de investigación

\section{INTRODUCCIÓN}

El porqué de una línea específica de investigación.

El objetivo de este trabajo es fundamentar posibles lineas de investigación que contribuyan al desarrollo de un modelo de aprendizaje de las ciencias por investigación a través de un estudio profundo y sistemático del uso del lenguaje en las disciplinas científicas. En nuestra actual legislación educativa se concede a la comprensión y producción de textos orales y escritos una importancia explícita cuando se alude, dentro de los objetivos generales de la Educación Secundaria Obligatoria a: «Comprender y producir mensajes orales y escritos con propiedad, autonomía y creatividad, adecuándolos a diferentes intenciones y contextos de comunicación y respetando otras formas de comunicación distintas a las babituales de su medio..."

Para lograr este objetivo es necesario, en primer lugar, conseguir que el profesorado, independientemente de la disciplina que imparte, comparta la responsabilidad de alcanzarlo. Ahora bien, a nuestro juicio, no basta un simple cambio actirudinal. Más allá de una descripción intuitiva y empírica de los problemas y su tratamiento también intuitivo y puntual, consideramos necesario aportar herramientas sistemáticas y bien fundamentadas para mejorar la comprensión y expresión de los alumnos en el ámbito concreto de cada área o disciplina. Ello justifica claramente, desde nuestro punto de vista, el desarrollo de una línea específica de investigación. 


\section{Hilo conductor de este trabajo}

Tras una primera clasificación de los problemas lingüísticos en el aprendizaje de las ciencias nos situaremos, teniendo en cuenta la evolución de la investigación educativa en este terreno, en la perspectiva de la construcción de textos dentro de la amplia variedad de situaciones comunicativas que tienen lugar en el aula, más allá de problemas puntuales tales como la mayor o menor precisión de la terminología empleada por los profesores, alumnos y autores de materiales didácticos.

La aproximación teórica a la estructura de los textos desde el modelo de Kintsch y Van Dijk y la discusión a partir del mismo de textos producidos por los alumnos en sus tareas escolares servirá para establecer de un modo más fundamentado la problemática en torno a la cual puede configurarse la linea de investigación cuyo interés pretendemos justificar. Partiendo de estas consideraciones, se lleva a cabo un amplio análisis descriptivo de los problemas que pueden identificarse en la producción de textos escritos por los alumnos. A partir de dicho análisis y teniendo en cuenta las concepciones teóricas actuales acerca de dicho proceso, intentaremos formular un conjunto de hipótesis de trabajo que puedan llegar a constituir un programa de investigación encaminado a mejorar el aprendizaje de las ciencias a través del progresivo perfeccionamiento de la comprensión y producción de textos escritos.

\section{MARCOTEÓRICO}

\section{Tres problemas linguiísticos de carácter general en el aprendizaje de las ciencias}

En un análisis a priori de los problemas lingüísticos en el aprendizaje de las ciencias, podemos señalar diferentes situaciones potencialmente problemáticas y ampliamente reflejadas en la bibliografía (Lloréns, 1988):

a) En primer lugar, el conflicto entre el carácter denotativo de la terminología científica en sí misma, y el carácter connotativo que en mayor o menor grado posee en su uso didáctico. Estos problemas derivan tanto de la evolución semántica de dichos términos, paralela al propio progreso científico, como de su polisemia, pues no podemos olvidar que, sobre todo en los niveles elementales, existe una elevada variabilidad semántica en el uso de los términos científicos en los contextos científicos y ordinarios que viene dada, en buena parte por las influencias socioculturales (Bensaude, 1982; Lloréns, 1987; Lloréns y Llopis, 1988; Lloréns y De Jaime, 1988).

b) En segundo lugar, la inadecuación entre los modos de pensamiento, capacidad de argumentación y estrategias de conceptualización de los niños y adolescentes y los rasgos ya señalados anteriormente del discurso científico, con sus implicaciones en la estructura global y morfosintaxis de los textos correspondientes. Este problema tiene dos grandes vertientes, por una parte, la relacionada con la expresión de conocimiento declarativo, propia de tareas como la elaboración de respuestas en pruebas escritas; por otra, la derivada de los procesos de discusión y reflexión colectiva característicos de una orientación constructivista del aprendizaje.

c) Por último, la dificultad, de índole metacognitiva, que supone para nuestros alumnos ser conscientes de la función de sus actividades dentro de su propio proceso de aprendizaje y que puede reflejarse en la inadecuación de los textos que producen a las situaciones comunicativas implícitas en el desarrollo del trabajo en clase. Este primer análisis general de los problemas lingüísticos en el aprendizaje de las ciencias puede servirnos para presentar de una manera ordenada el estado actual de la investigación en este terreno. 
En este sentido, cabe hacer alusión a un conjunto de investigaciones, muchas de ellas muy recientes, que orientan sus objetivos hacia los aspectos especificos del pensamiento científico, estudiando, bien a través del análisis del contenido de los manuales escolares (Eltinge y Roberts, 1993) bien a partir de los textos orales obtenidos mediante grabaciones en el aula, la formulación lingüística de las categorías del pensamiento científico y, particularmente, los procesos de cambio conceptual. Podemos citar, por ejemplo, la identificación de conductas relacionadas con aspectos del cambio conceptual (Basili y Sanford, 1991).para estudiar la eficacia del trabajo en pequeños grupos, el desarrollo en el aula de problemas prácticos (Watson, 1994) o las interacciones profesor-alumno en el desarrollo de tareas abiertas concebidas como investigaciones. Todas estas investigaciones estudian la estructura de los textos en relación a su función didáctica.

Contexto y co-texto: una visión sistémica de las situaciones de comunicación en el aula y, en particular, del papel de los textos escritos en dichas situaciones

Retomando las características de esta última linea de investigación, es importante distinguir en el análisis de cualquier texto utilizado o producido en el aula, el contexto y el co-texto (Castellá, 1992). El contexto posee según Schiffrin, D.(citado en Castellá, 1992) una triple dimensión: cognitiva (que se refiere a los conocimientos y experiencias del sujeto), cultural (que se refiere a los conocimientos y experiencias compartidos por una determinada comunidad) y social (definida por los distintos tipos de interacciones a través de ls cuales surge), y por otra, a su co-texto, es decir, al texto precedente $y$ al posterior.

Esta apreciación tiene importantes consecuencias didácticas: la consideración del contexto nos lleva a la necesidad de estudiar la producción de los textos por los alumnos y el modo de intervenir en su mejora considerando la intencionalidad didáctica y su relación con la organización del aprendizaje. Tener en cuenta el cotexto nos lleva a considerar la necesidad de estudiar dichos textos en el marco del programa de actividades, ya que su naturaleza depende de los textos orales y escritos que les anteceden o suceden. Es evidente, por ejemplo, que a la hora de precisar la estructura de un determinado informe sobre un trabajo práctico es imprescindible tener en cuenta qué tipo de texto escrito se utiliza como fundamentación teórica de referencia, qué características tienen nuestras instrucciones como texto oral, etc., así como la naturaleza de la reformulación o síntesis global a la que deseamos llegar a través de la discusión en clase de las conclusiones del trabajo práctico.

Centremos ahora nuestro análisis en los textos escritos producidos en el aula y detengámonos a considerar el cuaderno o diario del alumno. Éste es el documento que, en principio, debe constituir una reconstrucción del proceso de enseñanza/aprendizaje en definitiva, el curriculum real. También es el documento al que suele acudirse para estudiar o consultar. Estos textos suelen ser producidos, en muchas situaciones, a partir de la lectura de libros u otros documentos o bien a partir de textos orales: exposición del profesor, diálogo o intervenciones de compañeros. Los textos escritos también constituyen, en elevada proporción, el punto de partida sobre el que el profesorado evalúa el aprendizaje de sus alumnos, lo que implica un proceso de comprensión por parte de aquél. Asimismo, la comprensión de textos escritos, tanto procedentes de los materiales didácticos como de otras fuentes externas ocasionales -muchas veces debidamente adaptados- es un proceso habitual en la dinámica de la clase, del mismo modo que la producción de textos escritos por el profesorado (frecuentemente, como complemento de sus textos orales). Surge así, tal como se muestra en el siguiente diagrama, un complejo entrama- 
do de procesos de comprensión-producción de textos orales y escritos que caracterizan las diferentes situaciones comunicativas en el aula. Estos procesos suelen estar interrelacionados, de manera que probablemente será difícil estudiar o intervenir en uno de ellos sin tener en cuenta los restantes. Este enfoque sistémico no deberemos perderlo de vista tanto a la hora de analizar los problemas lingüísticos que surgen en ciertas situaciones como a la hora de prever los posibles obstáculos con que pueden enfrentarse nuestras iniciativas, así como para el diseño de la investigación.

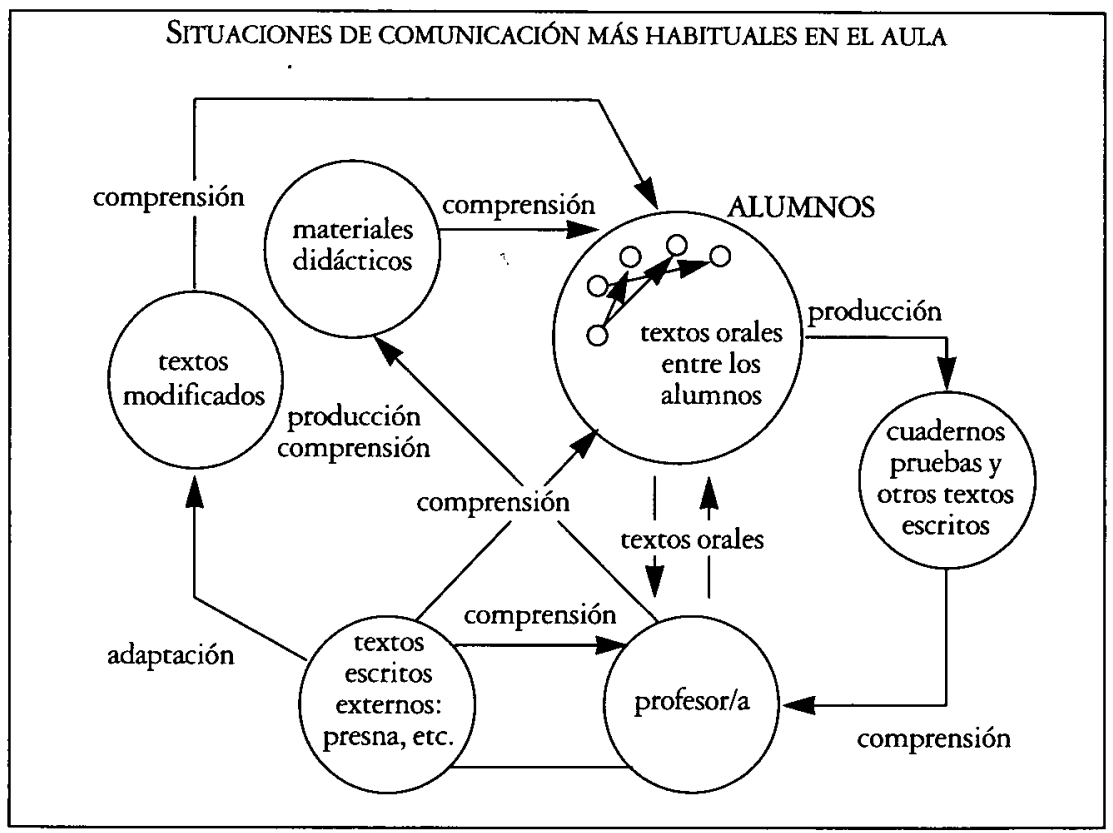

\section{Una aproximación teórica a la estructura de los textos científicos.}

El lenguaje científico posee ciertos rasgos específicos entre los que podemos destacar, en primer lugar, su carácter conceptual. La ciencia suele operar, más que con hechos u objetos, con clases de los mismos, es decir, con abstracciones. Por otra parte, estas abstracciones obedecen a un conjunto de criterios bien definidos, de manera que el lenguaje científico es eminentemente denotativo y tendente a la monosemia.

Al mismo tiempo, en la medida en que los procesos del pensamiento científico poseen ciertas características genuínas y están sujetos a las restricciones impuestas por la Lógica, los textos que entran en juego durante dichos procesos poseen unas características peculiares que afectan tanto a su estructura global, dando lugar a formas retóricas características, como a sus rasgos morfosintácticos. Desde el punto de vista de las tipologías textuales, partiendo del desarrollo de la propuesta de Werlich (Adam, 1985), los textos que aparecen en las clases de ciencias suelen ser de carácter explicativo y argumentativo, reflejando en sus rasgos estructurales aquellas operaciones cognitivas que constituyen un mayor obstáculo en el aprendizaje de los conceptos y procedimientos científicos (Van Dijk, 1989).

Para una aproximación fundamentada a dichos rasgos estructurales podemos acudir al modelo de Kintsch y Van Dijk para la comprensión de textos. Sánchez Miguel, E. (1989), entre otros autores, describe clara y detalladamente los diferentes aspectos 
de dicho modelo. Para los objetivos de nuestro trabajo haremos alusión tan sólo al carácter multiestructural de la representación del significado de un texto.

Desde este punto de vista podemos distinguir en una texto:

a) la microestructura, formada por las ideas elementales y su relación de continuidad entre ellas.

b) la macroestructura, constituida por aquellas ideas que dan coherencia global al texto.

c) la superestructura, que podría relacionarse con el tipo de texto, es decir, con su organización formal relacionada, al mismo tiempo, con su intención comunicativa.

La aplicación de estos conceptos a los textos construidos por los alumnos en sus tareas escolares puede servirnos para comenzar caracterizar y sistematizar sus problemas de expresión, constatando también la necesidad de partir de dos premisas básicas:

* La necesidad de referir cualquier aproximación a los problemas lingüísticos a los problemas específicos de la construcción de un determinado núcleo de conceptos.

* La necesidad de considerar el texto en el marco general del programa de actividades en que surge.

Considérese, por ejemplo, la respuesta a la siguiente cuestión:

Explicar por qué, al acercar una barra de PVC frotada con una piel de gato al péndulo eléctrico, se observa primero una atracción y después, una vez choca la bolita con la barra, una repulsión ¿Cómo averiguarías el signo de la carga que adquiere una barra de vidrio al ser frotada con seda?

*Al frotar el PVC con piel este se carga negativamente, al tocar la bolita la electricidad negativa se transmite a la bolita, entonces se atrae, pero al cargarse la bolita de electricidad negativa se repele el PVC puesto que es del mismo signo, entonces al acercar el vidrio cargado positivamente, puesto que lo bemos frotado con seda se separa del PVC y se pega al vidrio, dado que son de signos opuestos»

La actividad relacionada con esta pregunta trataba de plantear una situación problemática que exigiera poner en cuestión un modelo de carga eléctrica como propiedad general-macroscópica-de la materia, responsable de las atracciones y repulsiones entre distintos materiales frotados, para justificar la introducción de un primer modelo corpuscular de carga eléctrica que permitiera explicar los fenómenos de electrización por influencia. Hecha esta puntualización, analicemos la estructura de este texto distinguiendo el conjunto de ideas elementales que configurarían la microestructura del texto.

$P_{1}:$ El PVC frotado con piel adquiere carga negativa

$P_{2}$ : Cuando el $P V C$ toca la bolita le transmite electricidad negativa

$P_{3}:$ El PVC y la bolita se atraen

$P_{4}:$ La bolita se carga de electricidad negativa

$P_{\text {s: }}$ La bolita y el PVC se repelen

$P_{6}:$ La bolita y el $P V C$ son del mismo signo

$P_{7}$ : El vidrio está cargado positivamente

$P_{8}$ : El vidrio ha sido frotado con seda

$P_{9}$ : La bolita se separa del PVC

$P_{10}:$ La bolita se pega al vidrio

$P_{n}$ : La bolita y el vidrio se electrizan con cargas de signos opuestos

La macroestructura de este texro vendría dada por las ideas que contienen el significado global que da sentido a estos componentes locales. En este caso podríamos señalar:

$M_{1}$ : Diferentes materiales se electrizan con carga de distintos signo al ser frotados con otros materiales.

$M_{2}:$ Los objetos cargados eléctricamente pueden atraer objetos ligeros como la bolita de un péndulo eléctrico. 
$M_{3}$ : La carga puede transferirse por contacto.

$M_{4:}$ Los objetos cargados con el mismo signo se repelen y con signo contrario se atraen.

Utilizando la representación habitual de la relación entre microestructura y macroestructura podemos, en este caso, expresarla así:

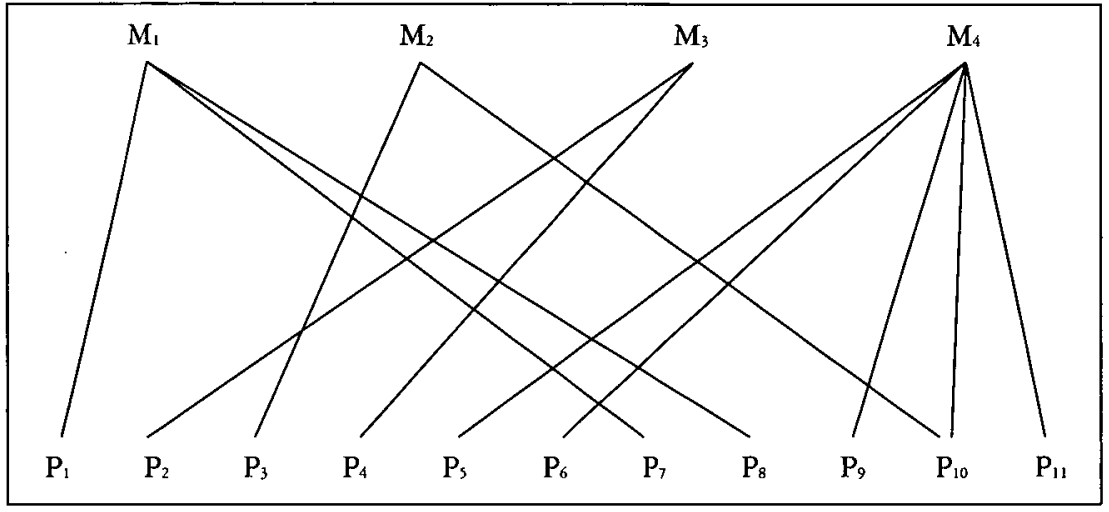

Detengámonos en primer lugar en cómo se produce la articulación de las ideas simples para obtener un conjunto de significados globales. En este texto, por ejemplo, se observa que el alumno sí conoce la asignación convencional de la carga - al PVC frotado con piel y de la carga + al vidrio frotado con seda, (aunque no explica dicho carácter convencional); asimismo, admite que existe una transferencia de carga, una "comunicación de esa propiedad", introducida de una manera intuitiva en clase -no se ha establecido el concepto de potencial eléctrico- ; en estas condiciones el alumno es capaz de elaborar un discurso correcto acerca del conjunto de atracciones y repulsiones que se observan. No obstante, la relación de causalidad que explica la atracción observada al principio no está bien definida y ello se debe, sin duda, a la ausencia de un modelo corpuscular de carga eléctrica que permita dar cuenta de la electrización por influencia (de hecho no existe una explicación de $\mathbf{M}_{2}$ )

Una respuesta adecuada acerca del fenómeno observado, dada por un alumno «experto» o su profesor implicaría la existencia de un conjunto de proposiciones elementales que reflejaran las cuatro siguientes macroproposiciones:

$N_{1}$ : Algunos fenómenos de electrización pueden explicarse admitiendo que los materiales, al frotarse, pueden adquirir una nueva propiedad general de la materia llamada carga eléctrica. Esta puede ser de dos tipos: positiva o negativa. Convencionalmente se asigna el signo negativo (electricidad "resinosa») a los objetos que se electrizan como el ámbar frotado con piel y positivo (electricidad "vitrea") a los que lo bacen como el vidrio frotado con seda.

$N_{2}$ : Los objetos electrizados con carga del mismo tipo se repelen y si lo son con carga de distinto signo se atraen.

$N_{3}$ : La materia puede considerarse constituida, en una primera aproximación, por partículas estáticas cargadas positivamente y un número igual de partículas negativas que pueden moverse libremente, de modo que la materia, en su conjunto, es eléctricamente neutra.

$N_{4}$ : Cuando se acerca un objeto cargado a uno que no lo está, las cargas negativas de éste pueden ser atraidas o repelidas, desplazándose y dando lugar en el segundo objeto, a una zona cargada positivamente y otra de carga negativa (electrización por influencia)

Obsérvese como estas macroproposiciones corresponden a los dos modelos teóricos para cuya transición sirve precisamente la actividad relacionada con el texto. $N_{1}$ correspondería a $M_{1}$ y $N_{2}$ a $M_{4}$, configurando el modelo teórico de partida que el 
alumno ya conoce. $\mathrm{N}_{3}$ y $\mathrm{N}_{4}$ reflejan el nuevo modelo teórico que permite explicar $\mathbf{M}_{2}$. Es importante hacer notar como la $\mathrm{M}_{3}$ sigue sin poder fundamentarse en una idea más general, ya que ello implicaría la extensión del modelo teórico incorporando los conceptos de campo y potencial eléctrico. Con esta discusión queremos ilustrar lo que, a nuestro juicio, es una importante premisa a la hora de fundamentar esta linea de investigación: creemos necesario situar el análisis de un texto o el establecimiento de criterios para su producción en el marco de los propios procesos de construcción de cada concepto científico, desconfiando, por tanto, de la eficacia de pautas de intervención generales aplicables al margen de un análisis específico desde el punto de vista de la propia naturaleza de los contenidos científicos.

Tengamos también presente, en segundo lugar, cómo, a la hora de valorar didácticamente los textos producidos por los alumnos, es imprescindible considerar la secuencia global de actividades, la cual determina la finalidad de aquellos. En este caso, la pregunta realizada tiene como objetivo situar al alumno frente a una situación problemática que exija cuya resolución justifique la necesidad de introducir un modelo corpuscular de carga eléctrica. Esta finalidad del texto, relacionada con su función didáctica y el nivel de formulación teórico en que se sitúa, implica una determinada forma organizativa o esquema tipológico: la denominada por Kinstch y Van Dijk superestructura. Así, en una actividad de este tipo, la superestructura podría esquematizarse así:

Establecimiento del marco teórico previo: explicación de las atracciones y repulsiones a partir del concepto de carga eléctrica como propiedad general de la materia.

Constatación de contradicciones o aspertos que no pueden explicarse: ¿por qué aparecen inetracciones entre objetos electrizados y otros que no lo están?

Descripción de bechos o experiencias que permiten profundizar o detallar más las contradicciones observadas: estudio del comportamiento del péndulo eléctrico.

Enunciado del problema, señalando como el marco teórico previo no puede explicarlo satisfactoriamente. La proximidad de objetos cargados puede inducir en objetos no cargados la aparición de una carga eléctrica de signo contrario, dando lugar a atracciones. Ello implica reconocer que ha habido una separación de cargas dentro del objeto. Un posible intento de solución puede ser considerar la relación entre carga y estructura de la materia, tratando así de explicar el proceso de electrización admitiendo el desplazamiento de partículas cargadas.

Sin embargo, si la pregunta se hubiera planteado como una actividad de evaluación para un estudiante que hubiera finalizado el estudio de la unidad didáctica, la superestructura podría venir dada por el esquema siguiente:

Establecimiento del marco teórico que da cuenta de todos los aspectos esenciales del fenómeno que pueden explicarse en el nivel correspondiente: modelo corpuscular de carga eléctrica e interpretación del principio de conservación de la carga. Explicación en estos tétminos de los procesos de electrización por frotamiento.

Establecimiento de restricciones en la aplicación del modelo teórico y proceso deductivo en el que van explicándose de manera jerarquizada las diferentes implicaciones del anterior marco teórico en los bechos estudiados: descripción en términos corpusculares de lo que ocurre en un objeto metálico no cargado cuando se le aproxima un objeto cargado y explicación de las atracciones y repulsiones que tienen lugar.

Identificación de los problemas abiertos que surgen como consecuencia de las insuficiencias del marco teórico previo: ¿cómo se explica la transferencia de carga eléctrica que existe al tomar contacto el péndulo con la barra cargada?

El análisis de este ejemplo puede servirnos para delimitar varios niveles a la hora de identificar los problemas lingüísticos que observamos en los textos que producen los alumnos:

a) los derivados de la cobesión intraproposicional, relacionados con la microestructura del texto. 
b) los derivados de la cobesión interproposicional, relacionados con la macroestructura del texto.

c) los derivados de la intencionalidad didáctica de la actividad, relacionados con la superestructura del texto.

El proceso de producción del texto: «decir el conocimiento» vs. «transformar el conocimiento»

Hasta aquí hemos contemplado la estructura del texto c̈ỏmo un producto acabado, posible objeto de análisis. Una aproximación más profunda al problema exige tener en cuenta el proceso de producción del texto desde el punto de vista teórico, ya que ello puede ayudarnos a comprender mejor algunos de los problemas que observamos, pudiendo apreciar así cómo tiene lugar la construcción de conocimientos a través del proceso de escritura. En este sentido debemos hacer referencia a la comparación propuesta por Scardamalia y Bereiter (1992) quienes proponen dos modelos explicativos de los procesos de producción de textos escritos: "decir el conocimiento» y «transformar el conocimiento». Dichos autores describen el proceso de maduración en la producción de textos escritos como la transición entre el modelo «decir el conocimiento», según el cual la producción del texto va produciéndose a medida que va recuperándose información de la memoria a través de un proceso de activación propagadora, en el que la coherencia del texto no depende de la aplicación deliberada o consciente de los conocimientos previos y de los objetivos del discurso (espacio retórico). Frente a este modelo, en el "transformar el conocimiento" el texto va generándose a partir de un proceso interactivo y dialéctico entre la información recuperada por la memoria y los conocimientos previos y la intencionalidad del texto. Se trataría, en definitiva, de la diferencia entre reproducir el conocimiento y refinar el texto; la diferencia podría expresarse también diciendo que el segundo aprende y construye conocimiento cuando escribe mientras el primero no.

\section{DESCRIPCIÓN DE LOS PROBLEMAS LINGÜÍSTICOS EN LA PRODUCCIÓN DE TEXTOS ESCRITOS DESDE UN ENFOQUE DEL APRENDIZAJE DE LAS CIENCIAS POR INVESTIGACION}

Tras todas estas reflexiones estamos en condiciones de abordar una clasificación de los problemas lingüísticos que favorezca el diseño de formas de intervención a través de las actividades del aula en el aprendizaje de las ciencias. La mayor parte de los problemas han sido identificados a través de la discusión de documentos de los alumnos en el marco de la experimentación de unidades didácticas en actividades de formación del profesorado en el CEP de Valencia y a lo largo de un curso monográfico de $3 .^{\circ}$ ciclo en el Departamento de Didáctica de las Ciencias Experimentales de la Universidad de Valencia.

Expondremos a continuación, de manera sistemática, estos problemas, según la siguiente secuencia, inspirada en el modelo de Flower y Hayes (Jolibert, 1992), según el cual la producción de un texto tiene lugar a través de las siguientes etapas: planificación, textualización y revisión.

1. Problemas derivadas del proceso global de producción del texto.

2. Problemas especificos del proceso de textualización:

a) en cuanto a la elaboración de la microestructura y macroestructura del texto.

b) en cuanto a la elaboración de la superestructura. 
Problemas derivados del proceso global de producción

Dentro de la etapa de planificación del texto podemos considerar diferentes aspectos que habitualmente suelen resultar problemáticos:

- Falta de adaptación al receptor (texto centrado en el emisor). El alumno no posee o no tiene presentes los conocimientos acerca de las posibles expectativas o conocimientos que posee el lector. Es habitual que los alumnos comiencen a escribir admitiendo implícitamente que el lector ya está inmerso en el tema y no necesita de ningún tipo de introducción; por ejemplo, cuando exponen argumentos dando por sobreentendido el conocimiento por parte del lector de la tesis que se pretende defender o refutar.

- Ausencia de un plan u objetivo global. Es muy corriente que los alumnos construyan el texto de una manera lineal, lo cual es muy coherente con el ya citado modelo: «decir el conocimiento», sin introducir en el texto proposiciones supraordenadas o materiales organizadores que muestren con claridad su hilo conductor $e$ intención.

- Falta de revisión o revisión inadecuada. En el mejor de los casos, cuando los alumnos revisan los textos que producen centran su atención en el vocabulario y la ortografía y rara vez introducen modificaciones en su contenido y su estructura.

\section{Problemas específicos del proceso de textualización:}

Durante el proceso de textualización hay problemas que se presentan de una manera generalizada y que pueden identificarse en cualquier tipo de texto, independientemente de su función didáctica. Tales problemas no son, generalmente, sino concreciones del modelo "decir el conocimiento".

Abordaremos en primer lugar aquellos problemas relacionados con la microestructura y macroestructura, es decir, a la cohesión intraproposicional y la interproposicional, respectivamente.

\section{Problemas relacionados con la microestructura y macroestructura}

Retomando la primera clasificación de los problemas lingüísticos, a un nivel de microestructura, considerando las informaciones discretas que son recuperadas de la memoria, podríamos citar diferentes factores que dificultan la cohesión intraproposicional. Desde el punto de vista léxico tenemos:

- La no distinción entre los significados cotidiano y científico de un término relativos a conceptos científicos.

- La no distinción del significado de un término dentro de diversos contextos científicos, por ejemplo, cuando existe una evolución semántica en el empleo de un determinado término en relación a la propia evolución de los conceptos científicos (calor, mol, etc.).

- El desconocimiento de ciertos atributos o aspectos del significado científico de un término.

- El empleo de términos «comodín» a nivel preconceptual, es decir, palabras que representan ideas vagas o difusas y que se utilizan indistintamente al referirse a distintos conceptos científicos.

- Excesiva utilización de palabras referidas a hechos y objetos concretos, no utilizando suficientemente los términos relativos a conceptos. Ello es especialmente significativo cuan- 
do se observa la escasa utilización espontánea de los términos correspondientes a las magnitudes físicas, lo que evidencia, consecuentemente, un deficiente nivel de generalización.

- Terminología específica de los procesos de la ciencia. Es un hecho ampliamente constatado la escasa e impropia utilización de la terminología relativa a los procesos de la ciencia -que se corresponde con un tratamiento epistemológico generalmente deficiente en los manuales escolares (Solaz, 1992)-

De las anteriores categorías pueden consultarse abundantes ejemplos, relacionados con los conceptos básicos de Química, en Lloréns (1989)

Desde el punto de vista sintáctico podemos referimos a:

- La tendencia a emplear oraciones innecesariamente largas y complejas.

- Los diferentes errores que dificultan una progresión temática u ordenación correcta de las ideas del texto, concretamente la paráfrasis, la aposición, la anáfora y la sustitución, así como el empleo deficiente de las concordancias.

- El empleo incorrecto o pobre de los tiempos verbales.

- Las deficiencias de todo tipo en la coordinación y subordinación. Este tipo de problemas suele trascender del ámbito microestructural, ya que suelen determinar el significado global de fragmentos importantes de texto, afectando, por lo tanto, a su macroestructura.

Las dificultades relacionadas con la cohesión interproposicional o macroestructural derivan en buena parte de las características del modelo: «decir el conocimiento». En este sentido debemos referirnos principalmente a:

- La elaboración lineal del texto sin jerarquización ni relaciones de supralsubordenación. Se corresponde con las dificultades de comprensión que se resumen en la expresión «tema + detalles», es decir, cuando la comprensión de un texto por parte del lector se reduce al recuerdo de su tema general y una colección de ideas referidas a dicho tema sin ningún tipo de vínculo jerárquico entre ellas. Asimismo, también se evidencia este problema en las dificultades que surgen al aplicar las macrorreglas cuando se elaboran resúmenes.

- El problema anterior suele observarse en los textos argumentativos, apareciendo frecuentemente una estructura como la siguiente: afirmación + justificaciones inconexas o no implicadas lógicamente con la afirmación, no existiendo una linea argumentativa clara.

- Como una consecuencia fácilmente interpretable desde el modelo «decir el conocimiento», se observan también numerosas incorporaciones de fragmentos y rasgos de textos de referencia (orales $u$ escritos) tal como son recuperados de la memoria, sin ser dialécticamente confrontados con los denominados: «espacio de contenido» y «espacio retórico»,-conceptos a los que ya nos referimos al describir el modelo de Scardamalia y Bereiter.

- También es corriente la incorporación al lenguaje escrito de formas típicas del lenguaje oral, tal como ocurre, por ejemplo, en las respuestas que comienzan así: « pues que...» y suelen ser textos a los que difícilmente se les puede atribuir un significado como tales, si no es a través de una referencia permanente al texto de la pregunta.

- La ausencia de conceptos estructurantes y procesos generales, impidiendo así, muchas veces, dotar al texto de mayor coherencia.

- Presencia de ideas irrelevantes que distraen al lector y dificultan la percepción de las ideas globales e importantes del texto. 
- Problemas de tipo sintáctico, relacionados con la estructura lógica del texto y el deficiente empleo, en general, de la conexión (Gardner, P.L., 1974, Maskill, R., 1988)

\section{Problemas relacionados con la superestructura del texto}

Ya hemos relacionado anteriormente la superestructura del texto con su funcionalidad didáctica. Por este motivo creemos adecuado, dentro de los objetivos de este trabajo, considerar previamente la naturaleza de las actividades más características de un enfoque del aprendizaje de las ciencias como investigación (Gil, 1993).

En la tabla siguiente hemos pretendido reflejar la relación entre los tipos de actividades citados, las principales características de los textos correspondientes y los problemas más habituales que suelen presentarse en las situaciones de aprendizaje. Estos problemas están relacionados con la superestructura del texto, aunque hemos extendido su descripción a aquellos problemas que, pese a corresponder a los niveles microestructurales o macroestructurales, son específicos de cada tipo de actividad. 


\begin{tabular}{|c|c|c|}
\hline FUNCION DIDACTICA & $\begin{array}{l}\text { PRINCIPALES CARACTERISTICAS } \\
\text { DE LOS TEXTOS PRODUCIDOS }\end{array}$ & $\begin{array}{c}\text { PROBLEMAS ESPECIFICOS } \\
\text { EN LA PRODUCCION DE ESTOS TEXTOS }\end{array}$ \\
\hline * Propuesta de objetivos. & $\begin{array}{l}\text { * Resúmenes elaborados a través de textos exposi- } \\
\text { tivos orales o escritos. } \\
\text { * Textos expositivos, en ocasiones con con una ele- } \\
\text { vada componente argumentativa, sobre proble- } \\
\text { mas cotidianos o posibles contradicciones o insu- } \\
\text { ficiencias que surgen al aplicar conocimientos } \\
\text { anteriores. } \\
\text { * Textos expositivos con abundante empleo de } \\
\text { categorías abstractas y metacognitivas. } \\
\text { * Textos muy diversos desde el punto de vista } \\
\text { tipológico, caracterizados por su relación con el } \\
\text { ámbito de lo afectivo. }\end{array}$ & $\begin{array}{l}\text { * Inexistencia de reelaboración lingüística: textos } \\
\text { que son un «collage» sin una estructura global, } \\
\text { que proviene de la ausencia de una lectura com- } \\
\text { prensiva de los materiales de referencia o de } \\
\text { exposiciones orales del profesor. } \\
\text { * No referencia a las fuentes de la información, si } \\
\text { ha lugar a ello. } \\
\text { * Pertinencia o no de las ideas expuestas (que reve- } \\
\text { la la comprensión o no de los aspectos esenciales } \\
\text { del problema investigado) } \\
\text { * Errores en las relaciones de supraordenación } \\
\text { entre conceptos. } \\
\text { * Fallos en la expresión del pensamiento causal, y } \\
\text { relaciones lógicas en general. } \\
\text { * Utilización de reglas de inferencia impropias del } \\
\text { pensamiento científico. } \\
\text { * Intentos de explicación que devienen en: } \\
\quad \text { - Meras descripciones. } \\
\text { - Afirmaciones apriorísticas: } \\
\text { * Inexistencia de argumentaciones objetivas. } \\
\text { * Expresión de las implicaciones personales de } \\
\text { modo poco creativo, recurriendo a estereotipos o } \\
\text { frases hechas. }\end{array}$ \\
\hline
\end{tabular}




\begin{tabular}{|c|c|c|}
\hline FUNCION DIDACTICA & $\begin{array}{l}\text { PRINCIPALES CARACTERISTICAS } \\
\text { DE LOS TEXTOS PRODUCIDOS }\end{array}$ & $\begin{array}{c}\text { PROBLEMAS ESPECIFICOS } \\
\text { EN LA PRODUCCION DE ESTOS TEXTOS }\end{array}$ \\
\hline \multicolumn{3}{|l|}{$\begin{array}{l}\text { 2. Establecimiento de estrategias para la reso- } \\
\text { lución de problemas. }\end{array}$} \\
\hline * Búsqueda de información. & $\begin{array}{l}\text { * Lectura comprensiva y elaboración de resúmenes } \\
\text { a partir de textos descriptivos o expositivos. } \\
\text { * Elaboración de informes: textos que implican la } \\
\text { reorganización y adaptación de información } \\
\text { diversa con objeto de adaptarla a las necesidades } \\
\text { de la actividad. }\end{array}$ & $\begin{array}{l}\text { * No adaptación a las exigencias de la actividad } \\
\text { que implica una comprensión del sentido que } \\
\text { posee. Falta de una visión global del propio pro- } \\
\text { ceso de aprendizaje. }\end{array}$ \\
\hline $\begin{array}{l}\text { * Expresión y puesta en común de conocimientos } \\
\text { previos. }\end{array}$ & $\begin{array}{l}\text { * Textos predominantemente argumentativos que } \\
\text { reflejan la respuesta a cuestiones concretas o bien } \\
\text { tienen como objetivo el debate entre compañe- } \\
\text { ros. } \\
\text { * Textos descritivos, tipo acta, que reflejan la } \\
\text { secuencia de argumentos aparecidos en un deba- } \\
\text { te en pequeño grupo o en la puesta en común } \\
\text { general. }\end{array}$ & $\begin{array}{l}\text { * Ausencia de lenguaje argumentativo. Simple } \\
\text { adición de ideas distintas e incluso, a veces, sin } \\
\text { que estas diferencias sean explícitias ni claras. } \\
\text { * Descripción del debate como yuxtaposición de } \\
\text { ideas individuales: no existe una diferencia cua- } \\
\text { litativa entre éstas y las ideas elaboradas por el } \\
\text { grupo como tal. Tampoco existe una relación } \\
\text { jerárquica entre argumentos sino un simple } \\
\text { encadenamiento lineal. }\end{array}$ \\
\hline & & $\begin{array}{l}\text { * No aparece o se muestra de manera confusa la } \\
\text { idea final o de consenso expresada por el grupo. }\end{array}$ \\
\hline
\end{tabular}




\begin{tabular}{|c|c|c|}
\hline FUNCION DIDACTICA & $\begin{array}{l}\text { PRINCIPALES CARACTERISTICAS } \\
\text { DE LOS TEXTOS PRODUCIDOS }\end{array}$ & $\begin{array}{c}\text { PROBLEMAS ESPECIFICOS } \\
\text { EN LA PRODUCCION DE ESTOS TEXTOS }\end{array}$ \\
\hline $\begin{array}{l}\text { 3. Actividades relacionadas con la formula- } \\
\text { ción y contrastación de hipótesis. }\end{array}$ & & \\
\hline * Formulación de hipótesis. & $\begin{array}{l}\text { * Enunciados característicos de tipo hipotético- } \\
\text { deductivo o enunciados declarativos de fuerte } \\
\text { componente abstracta. }\end{array}$ & $\begin{array}{l}\text { * Errores sintácticos que impiden la expresión de } \\
\text { las relaciones lógicas. }\end{array}$ \\
\hline * Establecimiento de planes de trabajo. & $\begin{array}{l}\text { * Textos expositivos con una elevada componente } \\
\text { predictiva que muestra claramente la relación } \\
\text { jerárquica entre las distintas etapas y aspectos de } \\
\text { la secuencia de trabajo. }\end{array}$ & $\begin{array}{l}\text { * No distinción entre las etapas o aspectos del tra- } \\
\text { bajo que van a desarrollarse simultaneamente y } \\
\text { las que guardan una secuencia temporal. }\end{array}$ \\
\hline $\begin{array}{l}\text { * Establecimiento de diseños experimentales y } \\
\text { secuencias operativas. }\end{array}$ & $\begin{array}{l}\text { * Textos eminentemente descriptivos e instructi- } \\
\text { vos, caracterizados estos últimos por una infor- } \\
\text { mación estructurada de manera lineal, no jerár- } \\
\text { quica, y la ausencia de un componente argumen- } \\
\text { tativo. }\end{array}$ & $\begin{array}{l}\text { * Secuenciación incorrecta o confusa. } \\
\text { * Imprecisión en el uso de términos específicos } \\
\text { sobre materiales o técnicas de trabajo. }\end{array}$ \\
\hline * Expresión de resultados. & $\begin{array}{l}\text { * Textos caracterizados por la utilización de esque- } \\
\text { mas formales: tablas, gráficos, etc. y por la } \\
\text { importancia del uso de las convenciones y sim- } \\
\text { bolismos. }\end{array}$ & $\begin{array}{l}\text { * Utilización de un lenguaje gráfico inconsistente } \\
\text { y no sujeto a criterios definidos. } \\
\text { * Utilización incorrecta o insuficiente de conven- } \\
\text { ciones o símbolos. No actualización de los mis- } \\
\text { mos. }\end{array}$ \\
\hline
\end{tabular}




\begin{tabular}{|c|c|c|}
\hline FUNCION DIDACTICA & $\begin{array}{l}\text { PRINCIPALES CARACTERISTICAS } \\
\text { DE LOS TEXTOS PRODUCIDOS }\end{array}$ & $\begin{array}{c}\text { PROBLEMAS ESPECIFICOS } \\
\text { EN LA PRODUCCION DE ESTOS TEXTOS }\end{array}$ \\
\hline $\begin{array}{l}\text { 4. Actividades de elaboración teórica. } \\
\text { * Discusión y análisis de los resultados. Delimita- } \\
\text { ción de su campo de validez. } \\
\text { * Establecimiento de conclusiones: } \\
\text { * Formulación de leyes y principios, estableci- } \\
\text { miento de relaciones con otros conceptos intra o } \\
\text { extradisciplinares, etc. } \\
\text { * Identificación de nuevos problemas. }\end{array}$ & $\begin{array}{l}\text { * Textos expositivos caracterizados por el predo- } \\
\text { minio de categorías abstractas y supraordenadas, } \\
\text { con una elevada exigencia de precisión en la ter- } \\
\text { minología empleada. } \\
\text { * Textos argumentativos generados a partir de la } \\
\text { evidencia de contradicciones o insuficiencias de } \\
\text { los conocimientos establecidos frente a nuevos } \\
\text { hechos experimentales o nueva información dis- } \\
\text { ponible. } \\
\text { En ambos casos también aparecerán: } \\
\text { * Textos predominantemente argumentativos que } \\
\text { reflejan la respuesta a cuestiones concretas o bien } \\
\text { tienen como objetivo el debate entre compañe- } \\
\text { ros. } \\
\text { * Textos descritivos, tipo acta, que reflejan la } \\
\text { secuencia de argumentos aparecidos en un deba- } \\
\text { te en pequeño grupo o en la puesta en común } \\
\text { general. }\end{array}$ & $\begin{array}{l}\text { * Falta de planificación del texto y, en consecuen- } \\
\text { cia, de una delimitación clara, desde los puntos } \\
\text { de vista conceptual y formal, del problema inves- } \\
\text { tigado, de los principales resultados obtenidos y } \\
\text { de las principales conclusiones elaboradas, así } \\
\text { como de los nuevos problemas derivados. } \\
\text { * Falta de precisión en el empleo del vocabulario } \\
\text { científico. }\end{array}$ \\
\hline
\end{tabular}




\begin{tabular}{|c|c|c|}
\hline FUNCION DIDACTICA & $\begin{array}{l}\text { PRINCIPALES CARACTERISTICAS } \\
\text { DE LOS TEXTOS PRODUCIDOS }\end{array}$ & $\begin{array}{c}\text { PROBLEMAS ESPECIFICOS } \\
\text { EN LA PRODUCCION DE ESTOS TEXTOS }\end{array}$ \\
\hline $\begin{array}{l}\text { 5. Actividades finales de una secuencia } \\
\text { de aprendizaje, utilizables como } \\
\text { evaluación. } \\
\text { * Síntesis. } \\
\text { * Actividades de consolidación de conocimientos a } \\
\text { través de su aplicación a nuevos contextos. } \\
\text { * Actividades de carácter metacognitivo o afectivo } \\
\text { para la evaluación de factores contextuales: clima de } \\
\text { la clase, organización de las tareas, etc. Elaboración } \\
\text { de diarios personales }\end{array}$ & $\begin{array}{l}\text { * Textos expositivos caracterizados por la presen- } \\
\text { cia de implicaciones personales y por la impor- } \\
\text { tancia de la estructura lógica y de la relación } \\
\text { jerárquica entre conceptos. } \\
\text { * Textos tipológicamente muy diversos cuya prin- } \\
\text { cipal exigencia puede ser la adecuada contextua- } \\
\text { lización a las nuevas situaciones en que se aplican } \\
\text { los conocimientos. Importancia también de los } \\
\text { textos relacionados con el trabajo en pequeños } \\
\text { grupos, cuyas características ya han sido citadas. } \\
\text { * Textos de elevado componente argumentativo y, } \\
\text { frecuentemente, con abundancia de categorías } \\
\text { relacionadas con lo afectivo y con diferentes } \\
\text { aspectos del proceso de aprendizaje. Pueden } \\
\text { adoptar la forma de textos amplios cuya estruc- } \\
\text { turación global la hace el alumno (diario) o bien } \\
\text { como respuestas cortas a cuestionarios (en cuyo } \\
\text { caso la estructuración global le viene dada) }\end{array}$ & $\begin{array}{l}\text { * Deficiente estructuración global, que puede con- } \\
\text { cretarse en: } \\
\text { - Ausencia de introducción. } \\
\text { - No delimitación de etapas, fases o aspec- } \\
\text { tos distintos. } \\
\text { - Ausencia de interrelaciones internas y } \\
\text { externas. } \\
\text { * Escaso nivel de apropiación del discurso, que } \\
\text { puede concretarse en: } \\
\text { - Ausencia de cualquier tipo de valoración } \\
\text { personal. } \\
\text { - Utilización de expresiones y vocabulario } \\
\text { poco personal o incorporado directamente } \\
\text { de las fuentes de información. } \\
\text { * Presencia de un lenguaje poco significativo, } \\
\text { cuando no meramente protocolario. }\end{array}$ \\
\hline
\end{tabular}


En la tabla anterior se utiliza el concepto de texto expositivo de la tipología de Werlich por su carácter más global en cuanto a las caracterísiticas generales de los textos científicos.

\section{POSIBLES LINEAS DE INVESTIGACIÓN E INNOVACIÓN}

La descripción que acabamos de exponer puede constituir la base de un programa de investigación encaminado a mejorar las producciones escritas de los alumnos en el aprendizaje de las ciencias. Siguiendo la estructura de dicha tipología, propondríamos tres líneas básicas de actuación, en cada una de las cuales podríamos formular una hipótesis central cuya operativización constituiría objeto de investigación. Estas lineas de investigación/innovación no sólo no serían excluyentes, sino que frecuentemente convergerían a la hora de definir las tareas escolares propuestas o los instrumentos de investigación.

En primer lugar, a la hora de identificar el problema básico objeto de investigación, cabría formular una hipótesis que podría enunciarse así:

El lenguaje utilizado por los alumnos en sus textos escritos presenta importantes deficiencias que afectan a la cohesión de los mismos y a su significado dentro del contexto de aprendizaje. Estas deficiencias pueden relacionarse con determinadas características del resto de textos orales y escritos que intervienen en las situaciones comunicativas del aula: exposiciones orales, materiales curriculares, etc., así como con la escasa sensibilidad del profesorado sobre este problema.

La operativización de esta hipótesis y su contrastación daría lugar a un conjunto de lineas de trabajo tales como:

- Análisis descriptivo de los problemas surgidos en los textos orales y escritos que surgen durante el desarrollo de la clase y estudio de sus interrelaciones.

- Estudio evolutivo a través de los diferentes niveles educativos de los problemas lingüísticos, así como el análisis diacrónico de los textos escritos producidos por un mismo alumno a lo largo de un periodo de su vida escolar.

- Estudio del pensamiento docente espontáneo acerca de los problemas lingüísticos: su grado de responsabilidad y competencia sobre los mismos y el tipo de recursos que pone en juego para resolverlos.

La contrastación de la hipótesis negativa debe traer consigo el diseño de investigaciones encaminadas a evaluar en el aula la eficacia de diferentes formas de intervención. Distingamos tres posibles campos de actuación:

\section{Producción de textos escritos por los alumnos}

\section{HIPÓTESIS FUNDAMENTAL:}

La instrucción en estrategias concretas de planificación del texto, textualización (mejora de la cohesión intraproposicional e interproposicional) y revisión producirá una mejora significativa en la capacidad de los alumnos para poner en juego procesos característicos de la metodología científica cuando se enfrentan a situaciones problemáticas en un contexto de aprendizaje por investigación.

La verificación de esta hipótesis implicaría la elaboración de una propuesta innovadora que incluiría, entre orras, las siguientes lineas de trabajo. 
- Desarrollo del vocabulario y su contextualización. Es necesario todavía profundizar en las conexiones semánticas -lo que se recupera en la memoria, es decir, el conocimiento que se activa al leer o escuchar ciertos términos-, así como establecer actividades para adecuada contextualización, fijando criterios para el uso preciso de la terminología científica.

- Desarrollo de los procesos de composición en el terreno sintáctico, a través de tareas específicas de construcción de frases y párrafos ( «sentence combining», por ejemplo, Daiker, 1982) y la aplicación en la clase de ciencias de estrategias para la mejora de la comprensión lectora (Sánchez Miguel, 1989)

- Desarrollo de esquemas apropiados para las distintas tareas específicas de la clase de ciencias. Aquí deberíamos incluir las estrategias de planificación de textos y el trabajo en clase con modelos textuales adecuados, así como el empleo de técnicas específicas (ordenación de frases, por ejemplo, -Vidal-Abarca, 1993)

\title{
Diseño de materiales didácticos.
}

\section{HIPÓTESIS FUNDAMENTAL}

\begin{abstract}
Una elaboración de los programas de actividades basada en criterios teóricamente fundamentados desde el punto de vista lingüístico dará lugar, además de una mejora en la comprensión de conceptos, una impacto positivo desde el punto de vista metacognitivo, favoreciendo una mejor comprensión por parte de los alumnos del significado de las actividades que realizan y de las ideas globales y estructurantes de los contenidos.
\end{abstract}

Entre las posibles lineas de trabajo derivadas de la operativización y contrastación de esta hipótesis podemos proponer el estudio de la influencia en el aprendizaje de variables relacionadas con la organización del texto y con el conocimiento de los términos relacionados con la metodología científica. Otro posible campo en el que podrían aportarse sensible mejoras en el diseño de materiales curriculares es en el estudio de la influencia de las llamadas actividades matemagénicas -actividades insertas en el proceso de lectura de un texto que favorecen su comprensión y contribuyen a construir conocimiento a partir del mismo- (Rothkopf, 1970)

\section{Actuación del profesorado}

\section{HIPÓTESIS FUNDAMENTAL}

\begin{abstract}
El desarrollo en el aula por parte del profesorado de pautas de actuación en sus interacciones verbales coherentes con las formas organizativas más adecuadas a los distintos aspectos del discurso científico, contribuirá a estructurar mejor los textos escritos producidos por los alumnos o las argumentaciones y otros textos orales que se desarrollan durante los debates y puestas en común.
\end{abstract}

La operativización y contrastación de esta hipótesis implicaría, por ejemplo, el análisis de grabaciones de audio y video de sesiones de trabajo en clase junto con el de los textos escritos: materiales curriculares, cuaderno de los alumnos y diario del profesor, tratando de identificar las superestructuras utilizadas en los diferentes textos y observando su evolución a lo largo de un periodo de tiempo en el cual se intentaran introducir por el profesorado determinadas pautas de interacción. 


\section{Referencias}

ADAM, J.M. (1985). ¿Quels types de textes? La français dans le monde, 192, 39-43.

AZANAR, E., Cros, A. QUINTANA, LL. (1991). "Coberencia textual y lectura". Barcelona, ICE/Horsori.

BARBA, R.H., (1993) A Study of Culturally Syntonic Variables in the Bilingual/Bicultural Science Classroom Joumal of Research in Science Teaching. Vol. 30, 9, pp. 1053-1071.

BENSAUDE, B. et al. (1982). Resultats d'une enquête sur quelques vocables d'orientation scientifique París, LIRESPT, E.N.S. Fontenay, E.N.S. Saint Cloud.

BrinCONES, I. y OTERO, J. (1994), Students' Conceptions of the Top-Level Structure of Physics Texts Science Education 78(2): 171-183.

CANDEla, A. (1993). «La construcción discursiva de la ciencia en el aula» Investigación en la Escuela, 21, 31-37.

CASSANY, D. (1991). Describir el escribir: cómo se aprende a escribir Barcelona, Paidós.

CARRETTO, J. et al. (1984). Enquête par questionnaires sur quelques vocables d'orientation scientifique Revue Française de Pedagogie, 68, 61-71.

CASSELS, J.R.T., 1976. Language in Chemistry: The effect of some aspects of language on "O" grade chemistry candidats Universidad de Glasgow. Unpublished M.S. Thesis.

CASSELS, J.R.T. y JOHNSTONE, A.H. (1977). Language in Chemistry (Research for the Classroom and Beyond. A report of Symposium. Universidad de Loughborough.

CASSELS, J.R.T. y JOHNSTONE, A.H. (1980) Understanding of Non-Technical Words in Science. Londres. Education Division. The Royal Society of Chemistry.

CASSELS, J.R.T. (1980) Language and Thinking in Science: some investigations with multiple choice questions. Universidad de Glasgow. Unpublished Ph.D. Thesis.

CAssels, J.R.T. y JoHNStone, A.H. (1983) The Meaning of Words and the Teaching Chemistry Education in Chemistry, 1983, 10-11.

Cassels, J.R.T. y JOHNSTONE, A.H. (1984) The Effect of Language on Student Performance on Multiple Choice Tests in Chemistry. Journal of Chemical Education. 61, 7, 613-614.

CASSELS, J.R.T. y JOHNSTONE, A.H. (1985) "Words that matter in Science. A report of a research exercise* Londres. Education Division of the Royal Society of Chemistry.

CASTELLÁ, J.M. (1992) «De la frase al text. Teories de l'ús lingüístic» Barcelona. Empúries.

CAZDEN, C.B. (1977) La situation: une source négligée des differences entre classes sociales dans l'utilisation du langage Universidad Paris XIII

Chardin, H., Vilain, C. y Giordan, A., 1986. «Un mot, un concept, le cas de l'immunologie Actas de las «jornadas sobre las técnicas de evaluación» Villa Arson, Nice.

Dillon, D.R., O'BRIEN, D.G.y MOJE, E.B. (1994). Literacy Learning in SEcondary School Science Classrooms: A Cross-Case Analysis of Three Qualitarive Studies Joumal of Rsearch in Science Teaching, 31, 4, 345-362.

Eltinge, E.M. y Roberts, C.W., (1993). Lingüistic Content Analysis: a Method to Measure Science as Inquiry in Textbooks. Joumal of Research in Science Teaching, 30,1, 65-84.

GARDNER, P.L. (1974) Language Difficulties of Science Studies The Australian Science Teachers Journal 20 1,63-76.

HANFLING, O. (1982) «Filosofía del lenguaje» I.C.E. Universidad de Valencia.

Herron, J.F. (1976) «Concept Formation as a Function of Institutional Procedure or: What Results from Innefective Teaching" Science Education 60(3), 375-388.

HER RON, J.D. (1977) «Are chemical terms well defined?» Journal of Chemical Education, 52, 758.

HER RON, J.D. (1979) «Hey, watch your language!» Journal of Chemical Education. 56, 330-331.

JOLIBERT, J. (1992) «Formar infants productors de textos» Ed. Graó, Barcelona.

LLORENS, J.A. y DE JAIME, C. (1987). "El medio cultural y la formación de los conceptos científicos» Infancia y Aprendizaje, 39-40, 47-55

LLORENS, J.A. y LLOPIS, R. y de Jaime, C. 1987. «El uso de la terminología científica en los alumnos que comienzan el estudio de la Química en la Enseñanza Media. Una propuesta metodológica para su análisis». Enseñanza de las ciencias, vol. 5, n. $^{\circ} 1$, pp. 33-40.

Markham, K.M., MinTzes, J.J. y GaIl, M., 1994. "The Concept Map as a Research and Evaluation Tool: Further Evidence of Validity". Joumal of Research in Science Teaching, 31, 1, 91-101.

POTH, W.M. , 1993. «Metaphors and Conversational Analysis as Tools in Reflection on Teaching Practice: Two Perspectives on Teacher-Student Interactions in Open-Inquiry Science» Science Education, $77,4,351-374$

ProverbIO, E. y LAI, S. 1987. "Le role du langage dans l'education scientifique: une tentative d'analyse fonctionelle Actas de las Jornadas de Educación Científica (JES) de Chamonix.

ROTH, W.M. y RoyCHOUdHuRY, A., (1993). «The Concept Map as a Tool for the Collaborative Construction of Knowledge: a Microanalysis of High School Physics Students" Joumal of Research in Science Teaching. 30, 5, 503-534.

ROTHKOPF, E.Z. 1970, "The Concept of Mathemagenic Activities». Review of Educational Research, 40, 325-336.

SÁNCHEZ Miguel, M. (1989). «Procedimientos para instruir en la comprensión de textos» Madrid, C.I.D.E.

SCARDAmalia, M. y BERÉlTER, C., (1984). «Development strategies in text processing» En H. Mandl, N. Stein y T. Trabasso (Ed.); Learning and comprebension of text. Hillsdale, N.J.: Erlbaum. 
Scardamalja, M. y Beréiter, C. (1992). "Dos modelos explicativos de los procesos de composición escrita», Infancia y Aprendizaje, 58, 43-63.

SERAFINI, M. 'T., (1989). «Cómo redactar un tema. Didáttica de la escritura *. Barcelona, Paidós.

SOLAZ, J.J. (1992) "El papel del texto en didáctica de las ciencias: una aproximación empírica a la comprensión lectora Trabajo de investigación de $3^{\circ}$ ciclo. Universidad de Valencia.

VAN DiJK, T. 1978. «La ciencia del texto. Un enfoque interdisciplinario» Barcelona, Paidós comunicación.

VAN DIJK, T. 1980.»Texto y contexto» Madrid, Cátedra.

VIDAL-ABARCA, E. y GILABERT, R. 1994, «Mapas de ideas: una herramienta para el aprendizaje escolar. Datos y comentarios para una discusión». Comunicación, lenguaje y educación, 21, 75-86.

VYGOTSKY, L.S. (1962) «Thought and Language» Cambridge, M.I.T. Press.

WATSON, J.R. 1994. «Students's engagement in practical problem solving: a case study", International Joumal of Science Education, 16, 1, 27-44.

\section{La producción de textos escritos en el aprendizaje de las ciencias. Bases para un programa de investigación Juan A. Lloréns y M. ${ }^{a}$ del Carmen Lorén CL\&E, 1995, 26}

Resumen: El artículo pretende establecer una primera clasificación de los problemas lingüísticos en el aprendizaje de las ciencias con el objetivo de situar al lector en la perspectiva de la construcción de textos dentro de la amplia variedad de situaciones comunicativas que tienen lugar en el aula, más allá de problemas puntuales tales como la mayor o menor precisión de la terminología empleada por los profesores, alumnos y autores de materiales didácticos.

La aproximación teórica a la estructura de los textos desde el modelo de Kintsch y Van Dijk y la discusión a partir del mismo de textos producidos por los alumnos en sus tareas escolares servirá para establecer de un modo más fundamentado la problemática en torno a la cual puede configurarse la linea de investigación cuyo interés pretendemos justificar. Partiendo de estas consideraciones, se lleva a cabo un amplio análisis descriptivo de los problemas que pueden identificarse en la producción de textos escritos por los alumnos. A partir de dicho análisis y teniendo en cuenta las concepciones teóricas actuales acerca de dicho proceso, intentaremos formular un conjunto de hipótesis de trabajo que puedan llegar a constituir un programa de investigación encaminado a mejorar el aprendizaje de las ciencias a través del progresivo perfeccionamiento de la comprensión y producción de textos escritos.

Datos sobre los autores: Juan A. Lloréns Molina es catedrático de educación secundaria en el I.B. "Juan de Garay» de Valencia y $\mathbf{M}^{\mathbf{a}}$ del Carmen de Jaime Lorén, profesora de Lengua y Literatura españolas en el I.F.P. «El Cabanyal» de Valencia.

Dirección: I.B. «Juan de Garay», C/Juan de Garay $n^{\circ}$ 27, 46014 Valencia.

( ) PERMISOS PARA CITAR O REPRODUCIR EN OTRAS FUENTES: Se pueden citar libremente hasta 500 palabras. Para reproducir una porción de texto mayor, figuras o ilustraciones, se deberá pedir permiso por escrito a la revista, especificando el uso al que se destina el texto. En todos los casos, se deberá citar el copyright de $C L \& E$. En el caso de artículos o textos que hayan sido a su vez reproducidos en $C L \& E$ los interesados deberán dirigirse tanto a los detentadores del copyright original como a $C L \& E$, en el caso de que se quiera hacer uso de la traducción. FOTOCOPIAS: Para todo lo relacionado con el uso mediante fotocopia del material de esta revista, deberán dirigirse a: CEDRO, C/ José Marañón, 10, 3. Izda. Tel. 5941575 . Fax 4453567 